\title{
UNIQUE LOCAL DETERMINATION OF CONVEX BODIES
}

\author{
E. Makai, JR., H. Martini
}

\begin{abstract}
Barker and Larman asked the following. Let $K^{\prime} \subset \mathbb{R}^{d}$ be a convex body, whose interior contains a given convex body $K \subset \mathbb{R}^{d}$, and let, for all supporting hyperplanes $H$ of $K$, the $(d-1)$-volumes of the intersections $K^{\prime} \cap H$ be given. Is $K^{\prime}$ then uniquely determined? Yaskin and Zhang asked the analogous question when, for all supporting hyperplanes $H$ of $K$, the $d$-volumes of the "caps" cut off from $K^{\prime}$ by $H$ are given. We give local positive answers to both of these questions, for small $C^{2}$-perturbations of $K$, provided the boundary of $K$ is $C_{+}^{2}$. In both cases, $(d-1)$-volumes or $d$-volumes can be replaced by $k$-dimensional quermassintegrals for $1 \leq k \leq d-1$ or for $1 \leq k \leq d$, respectively. Moreover, in the first case we can admit, rather than hyperplane sections, sections by $l$-dimensional affine planes, where $1 \leq k \leq l \leq d-1$. In fact, here not all $l$-dimensional affine subspaces are needed, but only a small subset of them (actually, a $(d-1)$-manifold), for unique local determination of $K^{\prime}$.
\end{abstract}

\section{$\S 1$ Introduction}

Barker and Larman [BL], p. 81, Conjecture 2, posed the following question. Let $K^{\prime} \subset \mathbb{R}^{d}$ be a convex body whose interior int $K^{\prime}$ contains a given convex body $K \subset \mathbb{R}^{d}$, and let, for all supporting hyperplanes $H$ of $K$, the areas, i.e., $(d-1)$ volumes of the intersections $K^{\prime} \cap H$ be given. Is then $K^{\prime}$ uniquely determined? The paper [BL] investigated only the case when $K$ is the unit ball $B^{d}$ and obtained several partial results to this question, for which we refer to their paper or, for some of them, cf. below. (There arises the question what happens if we replace hyperplane sections by sections by any affine $l$-planes $Y \subset \mathbb{R}^{d}$, for fixed $l \in[1, d-2]$, and we know the $l$-volumes of the intersections $K^{\prime} \cap Y$ for all $Y$ supporting $K$ - i.e., $Y$ intersects $K$ but not its interior. However, this would follow easily if we knew the result for hyperplanes. In fact, knowledge of all these $l$-volumes would determine the intersection of $K^{\prime}$ with any translate $Z+x$ of any linear $(l+1)$-subspace $Z$ of $\mathbb{R}^{d}$, such that $(Z+x) \cap(\operatorname{int} K) \neq \emptyset-$ hence also would determine $K^{\prime}$.)

The recent paper of Ryabogin-Yaskin-Zvavitch [RYZ] repeated this question in p. 332 as Question 8 , and its special case where $K=B^{d}$ in p. 331 as Question 7. The same was done in [YZh], in Problem 1.2 and Problem 1.3. Question 19 in [RYZ], p. 335 (that is, as they observe, equivalent to their Question 20, p. 336) is a special case of their Question 7. Namely, it asks the following. If $B^{d} \subset \operatorname{int} K^{\prime}$

1991 Mathematics Subject Classification. 2010 Mathematics Subject Classification. Primary: 52A2. Secondary: 52A38.

Key words and phrases. questions of Barker-Larman and Yaskin-Zhang, unique determination of convex bodies, quermassintegrals, radial function, star-shaped set, characterizations of symmetry. 
and the areas of the intersections of $K^{\prime}$ with any two parallel different tangent hyperplanes of $B^{d}$ are equal, is $K^{\prime}$ then 0 -symmetric? [RYZ] and [YZh] also give good overviews about results concerning questions of this type, as well as several new questions.

Yaskin-Zhang [YZh], Problem 1.4, posed the following question. Let $K^{\prime} \subset \mathbb{R}^{d}$ be a convex body whose interior contains a given convex body $K \subset \mathbb{R}^{d}$, and let, for all supporting hyperplanes $H$ of $K$, the $d$-volumes cut off from $K^{\prime}$ by the supporting hyperplanes $H$ of $K$ be given. Is then $K^{\prime}$ uniquely determined?

Barker-Larman [BL], Conjecture 1, which is repeated for the special case $K=B^{d}$ in [RYZ], p. 335, Question 18, is of similar type as those treated in this paper, but our methods do not yield its local solution. This question is: let $K \subset \operatorname{int} K^{\prime}$, where $K, K^{\prime} \subset \mathbb{R}^{d}$ are convex bodies with $K \subset$ int $K^{\prime}$, and let us have for each hyperplane $H$ supporting $K$ that $K^{\prime} \cap H$ is centrally symmetric. Is then $K^{\prime}$ centrally symmetric (for $K=B^{d} 0$-symmetric), or even an ellipsoid? Some considerations about this question see in $\S 2$, Problem. If $K$ is replaced by a point, e.g., $K=\{0\}$, and for some $l \in[2, d-1]$ and for each affine l-plane $P \ni 0$ we have that $K^{\prime} \cap P$ is centrally symmetric, then either $K$ is 0-symmetric, or $K$ is an ellipsoid. This is called the "false centre theorem", cf. [BL], p. 80. (There only the case $d-l=1$ is mentioned, but then, fixing $l$, a trivial induction for $d$ proves the above mentioned general result. Some further references to this problem are Aitchison-Petty-Rogers [APR], Larman [L], Montejano and Morales-Amaya [MM-A], Larman and Morales-Amaya [LM-A], V. Soltan [So], J. Jerónimo-Castro and T. B. McAllister [J-CM].)

We cite only some theorems. The first one is due to Santaló [Sa], see also [BL], Theorem 1: for $d=2$ and $K$ the unit disc with centre 0 , a concentric circle $K^{\prime}$ is uniquely determined. I.e., if all above sets $K^{\prime} \cap H$ (chords of $K^{\prime}$ tangent to $K$ ) have constant length, then $K^{\prime}$ is a circle with centre 0 . In fact, [Sa] proved this also for convex curves on $S^{2}$. This was reproved once more by Gorkavyy-Kalinin [GK], who also proved the analogous statement on the hyperbolic plane, and gave some further planar situations in which their proof works.

The next theorem is due to V. Yaskin [Y], Theorem: for $K$ the unit ball in $\mathbb{R}^{d}$, among convex polytopes, $K^{\prime}$ is uniquely determined. For dimension 2, a more general result was earlier proved by G. Xiong, Y.-w. Ma, W.-s. Cheung [XMCh], for $K 0$-symmetric and bd $K$, the boundary of $K$, "nice". I.e., if for two convex polytopes (polygons) $K_{1}^{\prime}, K_{2}^{\prime}$ containing $K$ in their interiors all above sets $K_{1}^{\prime} \cap H$ and $K_{2}^{\prime} \cap H$ (for $H$ being tangent hyperplanes of $K$ ) have equal areas (lengths), then $K_{1}^{\prime}=K_{2}^{\prime}$.

Next we cite [BL], Theorem 4, which answers the above question in the positive sense for $K=B^{d}$, when instead of hyperplane sections one considers sections with $l$-dimensional affine planes tangent to $B^{d}$ and their l-volumes, for fixed $l \in[1, d-2]$. However, observe that the supporting affine $l$-planes to any convex body $K \subset \mathbb{R}^{d}$ form an $((l+1)(d-l)-1)$-manifold (for $l=d-1$ a $(d-1)$-manifold), while

for $1 \leq l \leq d-2$ we have $(l+1)(d-l)-1>d-1$. 
The unknowns (values of the radial function for all $u \in S^{d-1}$ ) form a $(d-1)$ manifold. That is, intuitively one has for $l \in[1, d-2]$ "much more equations, namely $((l+1)(d-l)-1)$-manifold many" (the values of the $l$-volumes of all the sections of $K^{\prime}$ by supporting affine $l$-planes of $K$ ) "than unknowns, namely $(d-1)$ manifold many" (values of the radial function of $K^{\prime}$ ). This "explains solvability" of this problem for $K=B^{d}$ and $l \in[1, d-2]$, while the original problem with $K=B^{d}$ and $l=d-1$ is unsolved. In the statement of our Theorem 1 we eliminate this discrepancy between the dimensions of the respective manifolds.

The following theorem is due to [F] and [Ga83]: if for two distinct interior points $p_{1}, p_{2}$ of $K^{\prime}$ and any hyperplane $H$ containing any one of these points the area of the intersection $K^{\prime} \cap H$ is given, then $K^{\prime}$ is uniquely determined. Observe that here the hypothesis implies knowledge of the even parts $\left(\varrho_{i}^{d-1}(u)+\varrho_{i}^{d-1}(-u)\right) / 2$ of the functions $\varrho_{i}^{d-1}(u)$, where $\varrho_{i}(u)$ is the radial function of $K^{\prime}-p_{i}$, cf. [Gr], Theorem 5.6.3. That is, intuitively, we have "half information" both for $p_{1}$ and $p_{2}$, which together uniquely determine $K^{\prime}$. So here the heuristics works. As observed in [RYZ], p. 332, this statement is a variant [BL], p. 81, Conjecture 1 (cf. the first paragraph of the Introduction), when we replace the convex body $K$ by a non-degenerate segment. What happens if we replace $K$ by a non-empty compact convex set of fixed dimension in $[2, d-1]$ ? In particular, what happens if $K$ is replaced by a ball of the respective dimension?

The questions of Barker-Larman and Yaskin-Zhang seem to be difficult even for the plane with $K=B^{2}$. Rotating the sections $K^{\prime} \cap H$ by moving $u$ (the unit outer normal of $H$ ) in $S^{1}$ in the positive sense, and differentiating with respect to $u$ (for $\partial K$ being $C_{+}^{2}$ ), we obtain some equations containing the values and the first and second derivative values of some function at two different points, and unicity of the solution of the equation ought to be proved. So this way does not seem to lead to a solution.

We remark that an analogous question was settled by [MM] locally about the unit ball, under suitable smoothness hypotheses. That question was the following: if the $(d-2)$-dimensional surface area - or some other quermassintegral (or intrinsic volume) of smaller positive dimension - of the intersection of a convex body $K \subset \mathbb{R}^{d}$ with hyperplanes $u^{\perp}+\lambda u$, for any fixed $u \in S^{d-1}$, attains its maximum, e.g., at $\lambda=0$, is $K$ then 0 -symmetric? Here $u^{\perp}$ is the linear $(d-1)$-subspace orthogonal to $u \in S^{d-1}$. [MM], Theorem, settled this question, under suitable smoothness hypotheses, "locally", in the positive sense, close to the unit ball. More generally, hyperplanes can be substituted by sections with l-dimensional affine planes (and then we consider quermassintegrals of dimension in $[1, l]$ ), and still the analogous statement holds. The cases of l-volumes of sections with l-dimensional affine planes, for $l=1$ and $2 \leq l \leq d-1$, have been solved earlier in the positive sense, not only locally, but in their original form, by Hammer [H], Theorem 1, and Makai-MartiniÓdor [MMÓ], Corollary 3.2, respectively. Even, similarly to our Theorems 1 and 4 , it suffices to consider some family $\mathcal{L}_{l+1}$ of linear $(l+1)$-subspaces whose union is $\mathbb{R}^{d}$, and assume the maximality property only among translates of linear 
$l$-subspaces lying in some $L_{l+1} \in \mathcal{L}_{l+1}$ (cf. the first paragraph of the proof of our Theorem 1 and Remark 3). We will give in this paper, in the same spirit, "local solutions" to the questions of Barker-Larman and Yaskin-Zhang, cf. Theorems 1 and 4 below.

The questions of Barker-Larman and Yaskin-Zhang can be answered locally, close to any convex body of class $C_{+}^{2}$, not only to $B^{d}$. Let $K \subset \mathbb{R}^{d}$ be a $C_{+}^{2}$ convex body, with $0 \in \operatorname{int} K$, and let $K^{t} \supset K^{0}=K$ for $t \in[0,1]$ be a small $C^{2}$-perturbation of $K$. Here we consider $K^{t}$ to be given by its radial function $\varrho^{t}(u)$, for $u \in S^{d-1}$, and we investigate $\left.(\partial / \partial t) \varrho^{t}(u)\right|_{t=0}$, which is of course everywhere non-negative. Suppose that, for all tangent hyperplanes of $K$, either the "asymptotical behaviour" of the areas of their intersections with $K^{t}$, or the "asymptotical behaviour" of the volumes of the "caps" cut off from $K^{t}$ by them, for $t \rightarrow 0$, are given. Then "in first order" the approximation $K^{t}$ is uniquely determined.

I.e., suppose that there is a $C^{2}$-deformation $K^{t}$ of $K$, with $K^{t} \supset K^{0}=K$, for parameter values $t \in[0,1]$ — i.e., $[0,1] \times S^{d-1} \ni(u, t) \mapsto \varrho^{t}(u) \in(0, \infty)$ is a $C^{2}$ function. Suppose that we know either the "asymptotical behaviour" of the areas $\left((d-1)\right.$-volumes) of the intersection of each tangent hyperplane of $K$ with $K^{t}$, or the "asymptotical behaviour" of the $d$-volume of the "cap" cut off from $K^{t}$ by each tangent hyperplane of $K$, for $t \rightarrow 0$. (The exact meaning of "asymptotical behaviour" will be given in Theorems 1 and 4.) Then the first partial derivative of the radial function of $K^{t}$ with respect to $t$ at $t=0$, for each $u \in S^{d-1}$, is uniquely determined, cf. our Theorems 1 and 4 .

In both cases we may replace $(d-1)$-volumes and $d$-volumes by quermassintegrals (or intrinsic volumes) of any lower positive dimension $k$, and the analogous statements hold, cf. our Theorems 1 and 4.

Like in [MM], Theorem, and [MMÓ], Corollary 3.2, in our Theorem 1 we may allow, rather than sections by hyperplanes tangent to $K$, also sections with affine planes of lower, but positive dimension $l(\geq k)$, tangent to $K$, and the analogous statement holds, cf. our Theorem 1. This is a local positive answer to Question 1 of [YZh]. Here, however, recall from above that all tangent affine $l$-planes to $K$, which yield our equations about the "asymptotical behaviours", form an $((l+1)(d-l)-1)$-manifold, while the unknowns (values of the partial derivative of the radial function with respect to $t$, for $t=0$, and all $u \in S^{d-1}$ ) form a $(d-1)$-manifold, so that we have "much more equations than unknowns" (cf. (0.1) above). The particular case of our Theorem 1 , with $\mathcal{L}_{l+1}$ being the family of all linear $(l+1)$-subspaces of $\mathbb{R}^{d}$ which contain some fixed linear $l$-subspace of $\mathbb{R}^{d}$, uses only " $(d-1)$-manifold many" tangent affine $l$-planes to $K$ (cf. the paragraph before our Theorem 1), for the "( $d-1)$-manifold many" unknowns, and still has a positive answer. Thus also here the heuristics works.

Concerning convex bodies, we will use the standard notations, cf. [Sch]. We denote the norm of a vector $x \in \mathbb{R}^{d}$ by $\|x\|$. A convex body in $\mathbb{R}^{d}$ is a compact convex set in $\mathbb{R}^{d}$ with non-empty interior. The boundary and interior of a set $X \subset \mathbb{R}^{d}$ are denoted by bd $X$ and $\operatorname{int} X$, respectively. $V(\cdot)$ will denote ( $d$-dimensional) volume. 
We write conv $X$ and $\operatorname{lin} X$ for the convex hull and linear hull of a set $X \subset \mathbb{R}^{d}$, respectively. The unit ball and the unit sphere of $\mathbb{R}^{d}$ are denoted by $B^{d}$ and $S^{d-1}$, respectively. For the volume of $B^{d}$ we write $\kappa_{d}$. The quermassintegrals of non-empty compact convex sets $K$ (cf. [Sch], Ch. 4) are denoted by $W_{k}(K)$, for $0 \leq k \leq d$. Following [Sch], $\S 4.2$, we write $V_{k}(K):=\kappa_{d-k}^{-1}\left(\begin{array}{l}d \\ k\end{array}\right) W_{d-k}(K)$, which is called the $k$ 'th intrinsic volume of $K$, for $0 \leq k \leq d$. Then, by [Sch], 44.2 , for $r \in[0, \infty)$ we have $V\left(K+r B^{d}\right)=\sum_{i=0}^{d} \kappa_{d-k} V_{k}(K) r^{d-k}$. The intrinsic volumes $V_{k}(K)$ are monotonous, are positively homogeneous of degree $k$, and are continuous in the Hausdorff metric. Moreover, they remain unchanged if $\mathbb{R}^{d}$ is embedded in some higher dimensional Euclidean space, and we consider $V_{k}(K)$ as the $k$ 'th intrinsic volume of $K$ considered as a subset of the higher dimensional Euclidean space ([Sch], $\S 4.2$, p. 210; in fact, they are characterized by this property among constant multiples of $\left.W_{d-k}\right)$. By a star-shaped set, or star-shaped hull of a set we mean a star-shaped set, or star-shaped hull of a set with respect to 0 . The radial function of a compact star-shaped set $X \subset \mathbb{R}^{d}$ is $\varrho(\cdot): S^{d-1} \rightarrow[0, \infty)$, defined by $\varrho(u):=\max \{r \in[0, \infty) \mid r u \in X\}$. A convex body $K \subset \mathbb{R}^{d}$ is $C_{+}^{2}$ if its boundary is a $C^{2}$ submanifold of $\mathbb{R}^{d}$, with everywhere positive Gauss curvature. We write area for $(d-1)$-volume. If a convex body $K \subset \mathbb{R}^{d}$ is smooth and strictly convex, and $Y$ is a tangent affine plane of $K$ of some dimension, then we will write $\{y\}:=K \cap Y$, and the origin in $Y$ is chosen to be $y$. The Dupin indicatrix of a $C_{+}^{2}$ convex body $K \subset \mathbb{R}^{d}$, at some $x \in$ bd $K$, lying in the tangent hyperplane $H$ of $K$ at $x$, is obtained in the following way. Let in some fixed rectangular coordinate system with $x$ the origin and $H$ the hyperplane given by $x_{d}=0$, and with $K$ lying above $H$, bd $K$ have a local representation $x_{d}=f\left(x_{1}, \ldots, x_{d-1}\right)$. (Then $f(0, \ldots, 0)=0$ and, for each $i \in[1, d-1]$, also $f_{x_{i}}(0, \ldots, 0)=0$.) Then the Dupin indicatrix of $K$ at $x$, in the chosen rectangular coordinate system, is $\left\{\left(x_{1}, \ldots, x_{d-1}\right) \in H \mid(1 / 2) \sum_{i, j=1}^{d-1} f_{x_{i} x_{j}}(0, \ldots, 0) x_{i} x_{j}=1\right\}$.

\section{$\S 2$ THEOREMS}

Let $d \geq 2$, and $1 \leq k \leq l \leq d-1$ be any integers. Let $K \subset \mathbb{R}^{d}$ be a $C_{+}^{2}$ convex body, containing the origin in its interior, with radial function $\varrho(\cdot): S^{d-1} \rightarrow(0, \infty)$. Let, for $t \in[0,1]$, the convex body $K^{t}$ be a one-parameter deformation of $K$, with radial function $\varrho^{t}(\cdot): S^{d-1} \rightarrow(0, \infty)$, with $K^{t} \supset K^{0}=K$, and with $[0,1] \times S^{d-1} \ni$ $(t, u) \mapsto \varrho^{t}(u) \in(0, \infty)$ being a $C^{2}$ function. Then $\left.\left(\partial \varrho^{t}(u) / \partial t\right)\right|_{t=0}$ is, for each $u \in S^{d-1}$, non-negative. Let $\mathcal{L}_{l+1}$ denote a family of linear $(l+1)$-subspaces of $\mathbb{R}^{d}$, whose union is $\mathbb{R}^{d}$.

An example for $\mathcal{L}_{l+1}$ is the family of all linear $(l+1)$-subspaces of $\mathbb{R}^{d}$ which contain some fixed linear $l$-subspace of $\mathbb{R}^{d}$. Observe that this example forms a $(d-l-1)$-manifold. Then all affine $l$-planes, tangent to $K$, of these linear $(l+1)$ subspaces form a manifold of dimension $(d-l-1)+(l+1)-1=d-1$. For this example we have in the following Theorem 1 " $(d-1)$-manifold many equations" (one for each $Y$ in Theorem 1, namely for $\left.\lim _{\varepsilon \rightarrow 0} V_{k}\left(K^{\varepsilon} \cap L\right) / \varepsilon^{k / 2}\right)$, for " $(d-1)$-manifold 
many unknowns" (values of $\left.\left(\partial \varrho^{t}(u) / \partial t\right)\right|_{t=0}$, for all $\left.u \in S^{d-1}\right)$, which heuristically says that, according to the dimension of the manifolds in question, we have "as many equations as unknowns".

Theorem 1. Assume the hypotheses described before this theorem. Then the $k$ 'th intrinsic volume (e.g., the l-volume) of the intersection of $K^{\varepsilon}$ with each tangent affine l-plane $Y$ of $K$, divided by $\varepsilon^{k / 2}$, tends for $\varepsilon \rightarrow 0$ to a finite, non-negative limit, depending on $Y$. This limit as a function of $Y$, taken only for those $Y$ 's which lie in some linear $(l+1)$-subspace of $\mathbb{R}^{d}$ (namely in $\operatorname{lin} Y$ ) belonging to $\mathcal{L}_{l+1}$, uniquely determines $\left.\left(\partial \varrho^{t}(u) / \partial t\right)\right|_{t=0}$ for each $u \in S^{d-1}$.

Thus in the hypothesis we have information about the "asymptotical behaviour" of the intrinsic volumes of the intersections, and we have a conclusion about the "asymptotical behaviour" of the radial functions, for $\varepsilon \rightarrow 0$. The same holds for the intrinsic volumes of the "caps" cut off from $K^{\prime}$ in Theorem 4.

The statement for $1 \leq l \leq d-2$ is a relative of [BL], Theorem 4 . There a global uniqueness was proved, but only for $K=B^{d}$, and with all tangent affine $l$-planes of $K$.

Also in $[\mathrm{BL}]$, Theorem 5 , for $d$ odd, only a countably infinite set of values of $\varepsilon$ are used (sections by hyperplanes intersecting $B^{d}$, with distances from 0 in a countably infinite set). In our Theorem 1 this would "approximately" correspond to a series of $\varepsilon$ 's converging to 0 (actually, to different such sequences for different $Y$ 's) however, this does not substantially change the statement of our Theorem 1. The same holds for our Theorem 4.

The following Corollary 2 is a local version of [RYZ], p. 335, Question 19, in the same sense, as Theorem 1 is the local version of the question of Barker-Larman [BL], p. 81, Conjecture 2.

Corollary 2. Let all the hypotheses of Theorem 1 hold. Additionally, let $T: \mathbb{R}^{d} \rightarrow$ $\mathbb{R}^{d}$ be a linear isometry, such that $T K=K$. Let the $k$ 'th intrinsic volume (e.g., the l-volume) of the intersection of $K^{\varepsilon}$ with each tangent l-plane $Y$ of $K$, lying in some linear $(l+1)$-subspace of $\mathbb{R}^{d}$ (namely in $\operatorname{lin} Y$ ) belonging to $\mathcal{L}_{l+1}$, and with the tangent $l$-plane $T Y$ of $K$, lying in some linear $(l+1)$-subspace of $\mathbb{R}^{d}$ (namely in $T(\operatorname{lin} Y)$ ) belonging to $T\left(\mathcal{L}_{l+1}\right)$, divided by $\varepsilon^{k / 2}$, tend for $\varepsilon \rightarrow 0$ to the same limit. Then $\left.\left(\partial \varrho^{t}(u) / \partial t\right)\right|_{t=0}$ has equal values for $u$ and $T u$. In particular, for $T u=-u$ the function $\left.\left(\partial \varrho^{t}(u) / \partial t\right)\right|_{t=0}$ is an even function of $u \in S^{d-1}$.

Remark 3. Suppose the hypotheses of Theorem 1. Let, for $Y$ a tangent affine l-plane of $K, E^{Y}$ denote the Dupin indicatrix of $K \cap \operatorname{lin} Y$ at $y$ with $\{y\}=K \cap Y$. For unicity of $E^{Y}$ we suppose that the lengths of the vectors in the rectangular coordinate system in $\operatorname{lin} Y$ are equal to their lengths in the original polar coordinate system in $\mathbb{R}^{d}$. Then in Theorem 1 and Corollary 2 we can replace $V_{k}(\cdot)$ by any functions $F^{Y}(\cdot)$ (there being no compatibility conditions between the functions $F^{Y}(\cdot)$ for different $Y$ 's) which have the following properties.

1) They are defined on $[0, \infty) \times N^{Y}$, where $N^{Y}$ is some neighbourhood of the set conv $E^{Y}$ - considered in the set of convex bodies in $Y$ with the topology of the 
Hausdorff metric - and have values in $[0, \infty)$, but are positive on $(0, \infty) \times N^{Y}$. 2) They are positively homogeneous of degree $k$.

3) They are monotonous.

In particular, $F^{Y}(\cdot)$ (even defined for all non-empty compact convex subsets of $Y$ ) can be chosen as a mixed volume $V\left((\cdot), \ldots,(\cdot), K_{k+1}, \ldots, K_{l}\right)$, with $(\cdot)$ occurring $k$ times, and $K_{k+1}, \ldots, K_{l} \subset Y$ being any non-empty compact convex sets. (For mixed volumes cf. [Sch], Chapter 5.) But there are many other possibilities, e.g., the $k$ 'th intrinsic volume of the minimum volume circumscribed, or maximum volume inscribed ellipsoid, etc.

In the next theorem, a cap cut off from $K^{t}$ by a tangent hyperplane $H$ of $K$ is the intersection of $K^{t}$ and of the closed half-space $H^{+}$of $\mathbb{R}^{d}$, bounded by $H$ and not containing $K$. (Of course, here it makes no sense to consider affine planes of lower dimension.)

Theorem 4. Let all the hypotheses of Theorem 1 hold, with $l:=d-1$, but with $1 \leq$ $k \leq d$. Then the $k$ 'th intrinsic volume (e.g., the d-volume) of the cap cut off from $K^{\varepsilon}$ by each tangent hyperplane $H$ of $K$, divided by $\varepsilon^{k / 2}$ for $1 \leq k \leq d-1$, or by $\varepsilon^{(d+1) / 2}$ for $k=d$, respectively, tends for $\varepsilon \rightarrow 0$ to a finite non-negative limit, depending on $H$. This limit, as a function of $H$, uniquely determines $\left.\left(\partial \varrho^{t}(u) / \partial t\right)\right|_{t=0}$.

Observe that for $d=1$ (with $K, K^{\prime}$ then being segments with $K \subset K^{\prime}$ ) the function mapping $x \in \operatorname{bd} K$ to the volume (length) of the cap cut off from $K^{\prime}$ by $\{x\}$ trivially uniquely determines $K^{\prime}$. So we have not only a local, but a global solution. Therefore $d \geq 2$ is assumed in the hypotheses of Theorem 4 . The same holds also for the following Corollary 5.

Corollary 5. Let all the hypotheses of Theorem 4 hold. Additionally, let $T: \mathbb{R}^{d} \rightarrow$ $\mathbb{R}^{d}$ be a linear isometry such that $T K=K$. Let the $k$ 'th intrinsic volume (e.g., the $d$-volume) of the caps cut off from $K^{\varepsilon}$ by each tangent hyperplane $H$ of $K$, and by the tangent hyperplane $T H$ of $K$, divided by $\varepsilon^{k / 2}$ for $1 \leq k \leq d-1$, or by $\varepsilon^{(d+1) / 2}$ for $k=d$, respectively, tend for $\varepsilon \rightarrow 0$ to the same limit. Then $\left.\left(\partial \varrho^{t}(u) / \partial t\right)\right|_{t=0}$ has equal values for $u$ and $T u$. In particular, for $T u=-u$, the function $\left.\left(\partial \varrho^{t}(u) / \partial t\right)\right|_{t=0}$ is an even function of $u \in S^{d-1}$.

Remark 6. Suppose the hypotheses of Theorem 4, and let, for $H$ a tangent hyperplane of $K, E^{H}$ be as in Remark 3 (there $E^{Y}$ is written). Then in Theorem 4 and Corollary 5 we can replace $V_{k}(\cdot)$ by any functions $G^{H}(\cdot)$ (there being no compatibility conditions between the functions $G^{H}(\cdot)$ for different $H^{\prime}$ 's) which have the following properties.

1) They are defined on $[0, \infty) \times \tilde{N}^{H}$, where $\tilde{N}^{H}$ is some neighbourhood of the set conv $E^{H}$ - considered in the set of non-empty compact convex sets in $\mathbb{R}^{d}$, with the topology of the Hausdorff metric — and have values in $[0, \infty)$, but are positive on $(0, \infty) \times \tilde{N}^{H}$.

2) They are positively homogeneous of degree $k$.

3) They are monotonous.

4) They are continuous, from the restriction to $[0, \infty) \times \tilde{N}^{H}$ of the topology of the 
Hausdorff metric of non-empty compact convex sets in $\mathbb{R}^{d}$ to $[0, \infty)$. 5) If $k=d$, then still we have that $G^{H}$ is invariant under volume preserving affinities.

In particular, $G^{H}(\cdot)$ (even defined for all non-empty compact convex subsets of $\mathbb{R}^{d}$ ) can be chosen as a mixed volume $V\left((\cdot), \ldots,(\cdot), K_{k+1}, \ldots, K_{d}\right)$, with $(\cdot)$ occurring $k$ times, and $K_{k+1}, \ldots, K_{d} \subset \mathbb{R}^{d}$ being any non-empty compact convex sets. But there are many other possibilities, e.g., the $k$ 'th intrinsic volume of the minimum volume circumscribed, or maximum volume inscribed ellipsoid, etc.

Problem. The question of Barker-Larman [BL], p. 81, Conjecture 1, repeated in the special case $K=B^{d}$ in [RYZ], p. 335, Question 18, is the following. Let $K \subset$ $\operatorname{int} K^{\prime}$, where $K, K^{\prime} \subset \mathbb{R}^{d}$ are convex bodies, and let us have for each hyperplane $H$ supporting $K$ that $K^{\prime} \cap H$ is centrally symmetric. Is then $K^{\prime}$ centrally symmetric (for $K=B^{d} 0$-symmetric), or even an ellipsoid? (Of course, also here one could replace hyperplane sections by sections by any affine $l$-planes supporting $K$, for fixed $l \in[2, d-2]$, and suppose their central symmetry. However, a positive answer to [BL], p. 81, Conjecture 1, would easily imply the analogous statement for each $l \in[2, d-2]$. In fact, we can make an induction for $d-l$. Observe that by the false centre theorem, by our hypothesis, also the section of $K^{\prime}$ by each affine $(l+1)$-plane supporting $K$ is centrally symmetric.)

In our proof of Theorem 1 (cf. $\S 3$ ), the considered sections are in first approximation (that is, approximating bd $K^{\varepsilon}$ in the second order, up to terms of higher order) centrally symmetric with respect to the point of tangency (unique point of $K \cap Y$ ). Therefore, for a local variant of this question we would need to consider third order approximations of bd $K^{\varepsilon}$, of course assuming $C_{+}^{3}$. We could suppose that for these intersections there are some inner points (one can take, e.g., the barycentres) such that in opposite directions the radial functions associated to these points have values equal up to a factor $1+O\left(\varepsilon^{2}\right)$. The conclusion would be that $K^{\varepsilon}$ is $O\left(\varepsilon^{2}\right)$-close to some ellipsoid (and then $K$ would be exactly an ellipsoid). Is this true?

Returning to sections by all hyperplanes passing through some fixed point, there arises a related question: is there a stability variant of the false centre theorem?

\section{$\S 3$ Proofs}

Proof of Theorem 1 and of Remark 3. 1. It suffices to prove the case $l=d-1$. In fact, to calculate $\left.\left(\partial \varrho^{t} / \partial t\right)(u)\right|_{t=0}$ for $u \in S^{d-1}$, it suffices to consider some linear $(l+1)$-subspace in $\mathcal{L}_{l+1}$ containing $u$. There we can already calculate this quantity, using the case of $\mathbb{R}^{l+1}$ and affine $l$-planes in it.

2. Hence from now on we suppose $l=d-1$.

We give the proof for Theorem 1, which concerns the $k$ th intrinsic volume $V_{k}(\cdot)$. However, we will always stress (with italics or in brackets) what properties of $V_{k}(\cdot)$ are used, in order to see that the proof works also more generally for the functions $F^{Y}(\cdot)$ from Remark 3 .

In the whole proof, when the $\operatorname{sign} o(\cdot)$ is applied, it is meant for $\varepsilon \rightarrow 0$. We suppose in the whole proof that $\varepsilon>0$ is sufficiently small. 
Let $x \in \mathrm{bd} K$, and let us choose a rectangular coordinate system in $\mathbb{R}^{d}$ such that $x$ becomes the origin (thus we will have the radial function of $K$ with respect to some point of int $K$ ) and the lengths of the vectors in $\mathbb{R}^{d}$ in the original polar coordinate system and this rectangular coordinate system are identical. Further, the hyperplane $x_{d}=0$ should be a tangent hyperplane of $K$, with $K$ lying above this hyperplane.

Then bd $K$ can be given locally, close to $x=0$, as

$$
\left\{\begin{array}{l}
x_{d}=f\left(x_{1}, \ldots, x_{d-1}\right)=f(0, \ldots, 0)+\sum_{i=1}^{d-1} f_{x_{i}}(0, \ldots, 0) x_{i} \\
+(1 / 2) \sum_{i, j=1}^{d-1} f_{x_{i} x_{j}}(0, \ldots, 0) x_{i} x_{j}+o\left(x_{1}^{2}+\ldots+x_{d-1}^{2}\right) \\
=(1 / 2) \sum_{i, j=1}^{d-1} f_{x_{i} x_{j}}(0, \ldots, 0) x_{i} x_{j}+o\left(x_{1}^{2}+\ldots+x_{d-1}^{2}\right) .
\end{array}\right.
$$

Consider the outer normal of $K$ at $x=0$, and the point $x^{\varepsilon} \in \mathrm{bd} K^{\varepsilon}$ lying on this outer normal, below $x$ (i.e., on the negative $x_{d}$-axis, with 0 included). Let

$$
c(x):=-\left.(\partial / \partial t) f^{t}(0, \ldots, 0)\right|_{t=0}(\geq 0) .
$$

This number $c(x)$ can be expressed by the values of the function $[0,1] \times S^{d-1} \ni$ $(t, u) \mapsto \varrho^{t}(u) \in(0, \infty)$ and its first partial derivatives at $(0, x /\|x\|)$. We have to use the transition map between two coordinate systems, one being the polar coordinate system in $\mathbb{R}^{d}$, and the other one being the rectangular coordinate system introduced above. However, the explicit formula is not needed. Then non-negativity of $\partial \varrho^{t}(u) / \partial t$ for $t=0$, for each $u \in S^{d-1}$, is equivalent to non-negativity of $c(x)$, for any boundary point $x$ of $K$. From now on we will frequently write $\varepsilon$ rather than $t$, to emphasize its smallness. Observe that all boundary points $x^{\varepsilon}$ of $K^{\varepsilon}$ (which contains $K$ ) can occur here. Namely, for $x^{\varepsilon} \in \mathrm{bd} K^{\varepsilon}$ we can consider its image $x$ by the nearest point map $\mathbb{R}^{d} \backslash$ int $K \rightarrow$ bd $K$ : then $x^{\varepsilon}$ lies on the outward normal of $K$ at $x$.

Close to $x^{\varepsilon}$, bd $K^{\varepsilon}$ can be given locally as

$$
\left\{\begin{array}{l}
x_{d}=f^{\varepsilon}\left(x_{1}, \ldots, x_{d-1}\right)=f^{\varepsilon}(0, \ldots, 0)+\sum_{i=1}^{d-1} f_{x_{i}}^{\varepsilon}(0, \ldots, 0) x_{i} \\
+(1 / 2) \sum_{i, j=1}^{d-1} f_{x_{i} x_{j}}^{\varepsilon}(0, \ldots, 0) x_{i} x_{j}+o\left(x_{1}^{2}+\ldots+x_{d-1}^{2}\right) \\
=-(c(x)+o(1)) \varepsilon+O(\varepsilon) \sqrt{x_{1}^{2}+\ldots+x_{d-1}^{2}}+ \\
(1 / 2) \sum_{i, j=1}^{d-1} f_{x_{i} x_{j}}(0, \ldots, 0) x_{i} x_{j}+O(\varepsilon)\left(x_{1}^{2}+\ldots+x_{d-1}^{2}\right) \\
+o\left(x_{1}^{2}+\ldots x_{d-1}^{2}\right) .
\end{array}\right.
$$

In (1.3) we used that the values of the functions $f$ and $f^{\varepsilon}$ and their first and second derivatives with respect to $x_{1}, \ldots, x_{d-1}$ differ by at most $O(\varepsilon)(\mathrm{cf} .(1.1))$, and we used also (1.2). 
We will need these expansions only for the case when

$$
x_{1}^{2}+\ldots+x_{d-1}^{2}=O(\varepsilon)
$$

(the reason for this will be given later). So we suppose validity of (1.4) in the following.

By (1.4), (1.3) becomes

$$
x_{d}=f^{\varepsilon}\left(x_{1}, \ldots, x_{d-1}\right)=-(c(x)+o(1)) \varepsilon+(1 / 2) \sum_{i, j=1}^{d-1} f_{x_{i} x_{j}}(0, \ldots, 0) x_{i} x_{j}
$$

We have to consider the intersection $S^{\varepsilon}$ of $K^{\varepsilon}$ with the hyperplane $x_{d}=0$, and have to estimate its $k$ 'th intrinsic volume, for $1 \leq k \leq d-1$ ( $S$ for "section"). For some $\varepsilon_{0}>0$, for all $\varepsilon \in\left[0, \varepsilon_{0}\right]$ we have that $K^{\varepsilon}$ is $C_{+}^{2}$; we may suppose $\varepsilon_{0}=1$. Therefore either $x \in \operatorname{int} K^{\prime}$, and then $S^{\varepsilon} \cap\left(\operatorname{int} K^{\prime}\right) \neq \emptyset$, or $x \in \operatorname{bd} K^{\prime}$, and then $S^{\varepsilon}$ is the one point set consisting of the point of tangency of $K^{\varepsilon}$ with its tangent hyperplane given by $x_{d}=0$. Observe that in both these cases $S^{\varepsilon}$ is the convex hull of the intersection of bd $K^{\varepsilon}$ with the hyperplane $x_{d}=0$. For $x_{d}=0,(1.5)$ becomes

$$
(1 / 2) \sum_{i, j=1}^{d-1} f_{x_{i} x_{j}}(0, \ldots, 0) x_{i} x_{j}=(c(x)+o(1)) \varepsilon
$$

Thus we have to estimate the $k^{\prime}$ th intrinsic volume of $S^{\varepsilon}$, which set is the convex hull of the set given by (1.6), that is also the star-shaped hull of the set given by (1.6). This star-shaped hull is

$$
S^{\varepsilon}=\left\{\left(x_{1}, \ldots, x_{d-1}\right) \mid(1 / 2) \sum_{i, j=1}^{d-1} f_{x_{i} x_{j}}(0, \ldots, 0) x_{i} x_{j} \leq(c(x)+o(1)) \varepsilon\right\} .
$$

$$
\left\{\begin{array}{l}
\text { If } c(x)>0, \text { then let } 0<c_{1}<c(x)<c_{2} \text { be arbitrary. } \\
\text { If } c(x)=0, \text { then let } 0<c_{2} \text { be arbitrary. }
\end{array}\right.
$$

First we deal with the case $0<c(x)$. Then, for $\varepsilon>0$ sufficiently small, we have by (1.7)

$$
\left\{\begin{array}{l}
S_{1}^{\varepsilon}:=\left\{\left(x_{1}, \ldots, x_{d-1}\right) \mid \sum_{i, j=1}^{d-1}(1 / 2) f_{x_{i} x_{j}}(0, \ldots, 0) x_{i} x_{j} \leq c_{1} \varepsilon\right\} \\
\subset S^{\varepsilon}=\left\{\left(x_{1}, \ldots, x_{d-1}\right) \mid\right. \\
\left.(1 / 2) \sum_{i, j=1}^{d-1} f_{x_{i} x_{j}}(0, \ldots, 0) x_{i} x_{j} \leq(c(x)+o(1)) \varepsilon\right\} \\
\subset S_{2}^{\varepsilon}:=\left\{\left(x_{1}, \ldots, x_{d-1}\right) \mid(1 / 2) \sum_{i, j=1}^{d-1} f_{x_{i} x_{j}}(0, \ldots, 0) x_{i} x_{j} \leq c_{2} \varepsilon\right\}
\end{array}\right.
$$

We are going to give lower and upper estimates for $V_{k}\left(S^{\varepsilon}\right)$, cf. (1.12). For $0=c(x)$ 
the same considerations yield only the upper estimate for $V_{k}\left(S^{\varepsilon}\right)$ in (1.12): then we use the trivial lower estimate $0 \leq V_{k}\left(S^{\varepsilon}\right)$ rather than the one in (1.12) (cf. $(1.13))$.

We write

$$
E:=\left\{\left(x_{1}, \ldots, x_{d-1}\right) \mid(1 / 2) \sum_{i, j=1}^{d-1} f_{x_{i} x_{j}}(0, \ldots, 0) x_{i} x_{j} \leq 1\right\}
$$

Then $E$ is the convex hull of the Dupin indicatrix of $K$ at $x \in \mathrm{bd} K$, taken in our chosen rectangular coordinate system. By positive definiteness of the quadratic form $(1 / 2) \sum_{i, j=1}^{d-1} f_{x_{i} x_{j}}(0, \ldots, 0) x_{i} x_{j}$, the set $E$ is an 0 -symmetric ellipsoid. Then (1.9) can be rewritten as

$$
\left(c_{1} \varepsilon\right)^{1 / 2} E=S_{1}^{\varepsilon} \subset S^{\varepsilon} \subset S_{2}^{\varepsilon}=\left(c_{2} \varepsilon\right)^{1 / 2} E .
$$

Therefore even the third, i.e., largest set in (1.9) (and (1.11)) has a distance at most $O(\sqrt{\varepsilon})$ from 0 , so that we need to consider only such points $\left(x_{1}, \ldots, x_{d-1}\right)$, for which $x_{1}^{2}+\ldots+x_{d-1}^{2}=O(\varepsilon)$. This justifies the supposition of the validity of (1.4).

By $k$ 'th degree positive homogeneity and monotonicity of $V_{k}(\cdot),(1.11)$ implies

$$
\left\{\begin{array}{l}
0 \leq\left(c_{1} \varepsilon\right)^{k / 2} V_{k}(E)=V_{k}\left(\left(c_{1} \varepsilon\right)^{1 / 2} E\right) \leq V_{k}\left(S^{\varepsilon}\right) \leq \\
V_{k}\left(\left(c_{2} \varepsilon\right)^{1 / 2} E\right)=\left(c_{2} \varepsilon\right)^{k / 2} V_{k}(E) .
\end{array}\right.
$$

As mentioned just below (1.9), for $c(x)=0$ we have, rather than (1.12),

$$
0 \leq V_{k}\left(S^{\varepsilon}\right) \leq\left(c_{2} \varepsilon\right)^{k / 2} V_{k}(E)
$$

Hence for $0<c(x)$ by (1.12), while for $0=c(x)$ by (1.13), we have

$$
V_{k}\left(S^{\varepsilon}\right)=((c(x)+o(1)) \varepsilon)^{k / 2} V_{k}(E)
$$

for $\varepsilon \rightarrow 0$. Namely, for $0<c(x)$ we may choose both $c_{1}, c_{2}$ arbitrarily close to $c(x)$, and for $0=c(x)$ we may choose $c_{2}>0$ arbitrarily close to $0=c(x)$ (cf. (1.8)). We rewrite (1.14) as

$$
\lim _{\varepsilon \rightarrow 0} V_{k}\left(S^{\varepsilon}\right) /\left[\varepsilon^{k / 2} V_{k}(E)\right]=c(x)^{k / 2}
$$

By $(1.15) K$ and $\lim _{\varepsilon \rightarrow 0}\left(V_{k}\left(S^{\varepsilon}\right) / \varepsilon^{k / 2}\right)$, for each $x \in \operatorname{bd} K$, determine $c(x)$ uniquely. (Recall that $k \geq 1$.)

Last, taking into account (1.2), knowledge of this non-negative number $c(x)$, for each boundary point $x$ of $K$, determines the non-negative partial derivative of $\varrho^{t}(u)$ 
with respect to $t$, for $t=0$ and each $u \in S^{d-1}$. For this we have to use the values of the function $[0,1] \times S^{d-1} \ni(t, u) \mapsto \varrho^{t}(u) \in(0, \infty)$ and its first partial derivatives at $(0, x /\|x\|)$, and use the transition map between two coordinate systems: one is the polar coordinate system in $\mathbb{R}^{d}$, and the other one is the rectangular coordinate system used above in the proof.

Proof of Corollary 2. It follows immediately from Theorem 1. We only note that invariance of the first partial derivative of the perturbation with respect to $t$, for $t=0$, under the map $T$ in the rectangular coordinate systems at $x, T x \in \operatorname{bd} K$ used in the proof of Theorem 1, implies its invariance in the original polar coordinate system in $\mathbb{R}^{d}$ under the map $T$.

Proof of Theorem 4 and Remark 6. We give the proof for Theorem 4, which concerns the $k$ th intrinsic volume $V_{k}(\cdot)$. However, again we will always stress (with italics or in brackets) what properties of $V_{k}(\cdot)$ are used, in order to see that the proof works also more generally for the functions $G^{H}(\cdot)$ from Remark 6 .

We use the notations of the proof of Theorem 1. In particular, in the whole proof, when the sign $o(\cdot)$ is applied, it is meant for $\varepsilon \rightarrow 0$. Again we suppose in the whole proof that $\varepsilon>0$ is sufficiently small.

1. First we consider the case $k=d$, i.e., we consider the $d$-volume of the "caps" cut off from $K^{\varepsilon}$ by the tangent hyperplanes of $K$. We will write $V(\cdot)$ rather than $V_{d}(\cdot)$.

Till (1.8) we just use the considerations from the proof of Theorem 1.

Again, first we deal with the case $0<c(x)$.

In (1.9) we had inclusions of ("in general") $(d-1)$-dimensional compact convex sets in the hyperplane $x_{d}=0$. This has to be replaced by inclusions of ("in general") $d$-dimensional compact convex sets in $\mathbb{R}^{d}$.

We have to investigate the "cap" cut off from $K^{\varepsilon}$ by the tangent hyperplane $x_{d}=0$ of $K$, i.e., the set

$$
\left\{\begin{array}{l}
C^{\varepsilon}:=\left\{\left(x_{1}, \ldots, x_{d}\right) \mid f_{d}^{\varepsilon}\left(x_{1}, \ldots, x_{d-1}\right)=\right. \\
f^{\varepsilon}(0, \ldots, 0)+\sum_{i=1}^{d-1} f_{x_{i}}^{\varepsilon}(0, \ldots, 0) x_{i}+ \\
(1 / 2) \sum_{i, j=1}^{d-1} f_{x_{i} x_{j}}^{\varepsilon}(0, \ldots, 0) x_{i} x_{j}+o\left(x_{1}^{2}+\ldots+x_{d-1}^{2}\right) \leq x_{d} \leq 0
\end{array}\right.
$$

( $C$ for "cap"). We are going to give lower and upper estimates for $V\left(C^{\varepsilon}\right)$, cf. (4.7). For $0=c(x)$ the same considerations yield only (4.7) with omission of the middle expression, and replacing the equality sign by the $\leq$ sign. Then we use the trivial lower estimate $0 \leq V\left(C^{\varepsilon}\right)$ rather than the one derived from (4.5), second inequality. However, this gives the same formula (4.7), but omitting the second expression there, also for $0=c(x)$.

For $c(x)>0$ and $\varepsilon>0$ sufficiently small, taking into account (1.5), the set $C^{\varepsilon}$ in (4.1) contains 


$$
\left\{\begin{array}{l}
C_{1}^{\varepsilon}:=\left\{\left(x_{1}, \ldots, x_{d}\right) \mid-c_{1} \varepsilon+\right. \\
\left.(1 / 2) \sum_{i, j=1}^{d-1} f_{x_{i} x_{j}}(0, \ldots, 0) x_{i} x_{j} \leq x_{d} \leq 0\right\},
\end{array}\right.
$$

and is contained in

$$
\left\{\begin{array}{l}
C_{2}^{\varepsilon}:=\left\{\left(x_{1}, \ldots, x_{d}\right) \mid-c_{2} \varepsilon+\right. \\
\left.(1 / 2) \sum_{i, j=1}^{d-1} f_{x_{i} x_{j}}(0, \ldots, 0) x_{i} x_{j} \leq x_{d} \leq 0\right\} .
\end{array}\right.
$$

From (4.2) and (4.3) we have

$$
C_{1}^{\varepsilon} \subset C^{\varepsilon} \subset C_{2}^{\varepsilon} \text {. }
$$

Hence, by monotonicity and non-negativity of $V(\cdot)$, we have

$$
0 \leq V\left(C_{1}^{\varepsilon}\right) \leq V\left(C^{\varepsilon}\right) \leq V\left(C_{2}^{\varepsilon}\right) .
$$

$$
\left\{\begin{array}{l}
\text { For } i=1,2 \text { the sets } C_{i}^{\varepsilon} \text { are bounded by the }(d-1) \text {-ellipsoids } \\
S_{i}^{\varepsilon}(\text { cf. }(1.9)) \text { lying in the hyperplane given by } x_{d}=0, \\
\text { and by portions of the elliptic paraboloids given by } x_{d}= \\
-c_{i} \varepsilon+(1 / 2) \sum_{i, j=1}^{d-1} f_{x_{i} x_{j}}(0, \ldots, 0) x_{i} x_{j}, \text { lying below } S_{i}^{\varepsilon}
\end{array}\right.
$$

The volume of $C_{i}^{\varepsilon}$ can be calculated as $2 /(d+1)$ times the volume of its circumscribed right cylinder $\left(C_{i}^{\varepsilon}\right)^{\prime}$, with upper base the ellipsoid $S_{i}^{\varepsilon}$ and height $c_{i} \varepsilon$. In fact, by applying an affinity we may suppose $E=B^{d-1}$ and $c_{i} \varepsilon=1$, and then we calculate $V\left(C_{i}^{\varepsilon}\right) / V\left(\left(C_{i}^{\varepsilon}\right)^{\prime}\right)$ by using polar coordinates in the hyperplane given by $x_{d}=0$. (For the case of $G^{H}(\cdot)$ we observe that this affinity can be factorized as the product of a magnification in a positive ratio, and a volume-preserving affinity.) Then for $\varepsilon>0$ sufficiently small, analogously to (1.14) we have, both for $0<c(x)$ and for $0=c(x)($ for $0=c(x)$ omitting the middle term)

$$
\left\{\begin{array}{l}
V\left(C^{\varepsilon}\right)=\left[\kappa_{d-1} /\left[\operatorname { d e t } \left(( 1 / 2 ) \left(f_{x_{i} x_{j}}(0, \ldots, 0)\right.\right.\right.\right. \\
\left./((c(x)+o(1)) \varepsilon)))]^{1 / 2}\right] \cdot((c(x)+o(1)) \varepsilon) \cdot 2 /(d+1)= \\
{\left[\kappa_{d-1} /\left[\operatorname{det}\left((1 / 2) f_{x_{i} x_{j}}(0,0, \ldots, 0)\right)\right]^{1 / 2}\right] \cdot[2 /(d+1)] .} \\
((c(x)+o(1)) \varepsilon)^{(d+1) / 2} .
\end{array}\right.
$$

(For Remark 6 we do not have (4.7), since we do not know $G^{H}(E)$. First suppose 
$0<c(x)$. Then we have the analogue of (4.5) with $G^{H}$, and by (4.6) and (1.8) we get $G^{H}\left(\left(C_{1}^{\epsilon}\right)^{\prime}\right) / G^{H}\left(\left(C_{2}^{\epsilon}\right)^{\prime}\right)=1+o(1)$. Therefore, we have the analogue of $(4.7)$, namely $G^{H}\left(C^{\epsilon}\right)=G^{H}\left(E^{\prime}\right) \cdot((c(x)+o(1)) \varepsilon)^{(d+1) / 2}$. Here $E^{\prime}$ is the set bounded by $E$ (defined in (1.10)) and by the portion of the elliptic paraboloid given by $x_{d}=-1+(1 / 2) \cdot \sum_{i, j=1}^{d-1} f_{x_{i} x_{j}}(0, \ldots, 0) x_{i} x_{j}$, lying below $E$. The case $0=c(x)$ is treated like in the case of $V(\cdot)$, only using $G^{H}(\cdot)$ rather than $V(\cdot)$.)

Hence, analogously to (1.15), we have from (4.7)

$$
\left\{\begin{array}{l}
\lim _{\varepsilon \rightarrow 0} V\left(C^{\varepsilon}\right) \cdot\left[\operatorname{det}\left((1 / 2)\left(f_{x_{i} x_{j}}(0, \ldots, 0)\right)\right)\right]^{1 / 2} / \\
{\left[\kappa_{d-1}[2 /(d+1)] \varepsilon^{(d+1) / 2}\right]=c(x)^{(d+1) / 2}}
\end{array}\right.
$$

By (4.8) $K$ and $\lim _{\varepsilon \rightarrow 0}\left(V\left(C^{\varepsilon}\right) / \varepsilon^{(d+1) / 2}\right)$, for each $x \in$ bd $K$, determine $c(x)$ uniquely. (For Remark 6, we use here once more $G^{H}\left(E^{\prime}\right)$, like below (4.7).) Then the repetition of the last paragraph of the proof of Theorem 1 finishes the proof for the case $k=d$.

2. We turn to the case $1 \leq k \leq d-1$. Again, first we deal with the case $0<c(x)$.

For $0=c(x)$ the same considerations yield for $V_{k}\left(C^{\varepsilon}\right)$ only (4.17). Then we use the trivial lower estimate (4.11) rather than (4.10). However, this gives the same formula (4.18), also for $0=c(x)$.

By (1.11) we have $\left(c_{1} \varepsilon\right)^{1 / 2} E=S_{1}^{\varepsilon} \subset S^{\varepsilon} \subset C^{\varepsilon}$. Here for $\varepsilon \rightarrow 0$ we may choose $0<c_{1}=c(x)+o(1)\left(\text { cf. (1.8)), hence } C^{\varepsilon} \supset((c(x)+o(1))) \varepsilon\right)^{1 / 2} E$. Thus, by $k^{\prime}$ th degree positive homogeneity and monotonicity of $V_{k}(\cdot)$, we have

$$
V_{k}\left(C^{\varepsilon}\right) /\left[V_{k}(E) \cdot \varepsilon^{k / 2}\right] \geq(c(x)+o(1))^{k / 2} .
$$

For $0=c(x)$ we use the trivial estimate

$$
V_{k}\left(C^{\varepsilon}\right) /\left[V_{k}(E) \cdot \varepsilon^{k / 2}\right] \geq 0
$$

We turn to the upper estimate. Recall that $c_{2}>0$ (cf. (1.8)). With $\left(C_{2}^{\varepsilon}\right)^{\prime}$ as defined below (4.6) we have by (4.4) $C^{\varepsilon} \subset C_{2}^{\varepsilon} \subset\left(C_{2}^{\varepsilon}\right)^{\prime}$. By monotonicity of $V_{k}(\cdot)$ this implies $V_{k}\left(C^{\varepsilon}\right) / V_{k}\left(\left(C_{2}^{\varepsilon}\right)^{\prime}\right) \leq 1$. Equivalently, using $k$ 'th degree positive homogeneity of $V_{k}(\cdot)$, we have

$$
V_{k}\left(C^{\varepsilon} /\left(c_{2} \varepsilon\right)^{1 / 2}\right) / V_{k}\left(\left(C_{2}^{\varepsilon}\right)^{\prime} /\left(c_{2} \varepsilon\right)^{1 / 2}\right) \leq 1
$$

Now observe that $\left(C_{2}^{\varepsilon}\right)^{\prime} /\left(c_{2} \varepsilon\right)^{1 / 2}$ is a right cylinder with base $S_{2}^{\varepsilon} /\left(c_{2} \varepsilon\right)^{1 / 2}=E$ (cf. (1.11)) and height $\left(c_{2} \varepsilon\right) /\left(c_{2} \varepsilon\right)^{1 / 2}=\left(c_{2} \varepsilon\right)^{1 / 2}$. Therefore $\left(C_{2}^{\varepsilon}\right)^{\prime} /\left(c_{2} \varepsilon\right)^{1 / 2}$ is in the $\left(c_{2} \varepsilon\right)^{1 / 2}$-neighbourhood of $E$.

Therefore we have, by continuity of $V_{k}(\cdot)$, that 


$$
\left.V_{k}\left(C_{2}^{\varepsilon}\right)^{\prime} /\left(c_{2} \varepsilon\right)^{1 / 2}\right) / V_{k}(E) \leq 1+o(1)
$$

Multiplying (4.14) and (4.15) we get

$$
V_{k}\left(C^{\varepsilon} /\left(c_{2} \varepsilon\right)^{1 / 2}\right) / V_{k}(E) \leq 1+o(1) .
$$

Since we may choose $c_{2}>0$ arbitrarily close to $c(x)$ (cf. (1.8)), therefore once more by $k$ 'th degree positive homogeneity of $V_{k}(\cdot),(4.16)$ implies

$$
V_{k}\left(C^{\varepsilon}\right) /\left[V_{k}(E) \cdot \varepsilon^{k / 2}\right] \leq c_{2}^{k / 2}(1+o(1))=(c(x)+o(1))^{k / 2} .
$$

Then for $0<c(x)$ (4.10), while for $0=c(x)$ (4.11), together with (4.17) give

$$
V_{k}\left(C^{\varepsilon}\right) /\left[V_{k}(E) \cdot \varepsilon^{k / 2}\right]=(c(x)+o(1))^{k / 2} .
$$

We rewrite (4.18) as

$$
\lim _{\varepsilon \rightarrow 0} V_{k}\left(C^{\varepsilon}\right) /\left[V_{k}(E) \cdot \varepsilon^{k / 2}\right]=c(x)^{k / 2} .
$$

By (4.19) $K$ and $\lim _{\varepsilon \rightarrow 0}\left(V_{k}\left(C^{\varepsilon}\right) / \varepsilon^{k / 2}\right)$, for each $x \in \operatorname{bd} K$, determine $c(x)$ uniquely. (Recall that $k \geq 1$.)

Then the repetition of the last paragraph of the proof of Theorem 1 finishes the proof for the case $1 \leq k \leq d-1$.

Proof of Corollary 5. It follows immediately from Theorem 4, also taking into account the proof of Corollary 2.

\section{REFERENCES}

[APR] P. W. Aitchison, C. M. Petty, C. A. Rogers, A convex body with a false centre is an ellipsoid, Mathematika 18 (1971), 50-59. MR 45\#2572.

[BL] J. A. Barker, D. G. Larman, Determination of convex bodies by certain sets of sectional volumes, Discrete Math. 241 (2001), 79-96. MR 2002j:52001.

[F] K. Falconer, X-ray problems for point sources, Proc. London Math. Soc. (3) 46 (1983), 241-262. MR 85g:52001a.

[Ga83] R. J. Gardner, Symmetrals and X-rays of planar convex bodies, Arch. Math. (Basel) 41 (1983), 183-189. MR 85c:52007.

[Ga] R. J. Gardner, Geometric Tomography; Second edition, Encyclopedia of Math. and its Appl. 58, Cambridge Univ. Press, Cambridge, 1995; 2006. MR 96j:52006; 2007i:52010.

[GK] V. Gorkavyy, D. Kalinin, Barker-Larman problem in the hyperbolic plane, Results Math. 68 (2015), 519-525. MR 3407571. 
[Gr] H. Groemer, Geometric Applications of Fourier Series and Spherical Harmonics, Encyclopedia of Math. and its Appl. 61, Cambridge Univ. Press, Cambridge, 1996. MR 97j:52001.

[H] P. C. Hammer, Diameters of convex bodies, Proc. Amer. Math. Soc. 5 (1954), 304-306. MR 15,819b.

[J-CM] J. Jerónimo-Castro, T. B. McAllister, Two characterizations of ellipsoidal cones, J. Convex Anal. 20 (2013), 1181-1187. MR 3184302.

[L] D. G. Larman, A note on the false centre theorem, Mathematika 21 (1974), 216-227. MR 50\#14490.

[LM-A] D. G. Larman, E. Morales-Amaya, On the false pole problem, Monatsh. Math. 151 (2007), 271-286. MR 2008d:52006.

[MM] E. Makai, Jr., H. Martini, Centrally symmetric convex bodies and sections having maximal quermassintegrals, Studia Sci. Math. Hungar. 49 (2012), 189-199. MR 3058386.

[MMÓ] E. Makai, Jr., H. Martini, T. Ódor, Maximal sections and centrally symmetric convex bodies, Mathematika 47 (2000), 17-30. MR 2003e:52005.

[MM-A] L. Montejano, E. Morales-Amaya, Variations of classic characterizations of ellipsoids and a short proof of the false centre theorem, Mathematika 54 (1-2) (2007), (1-2), 35-40. MR 2009c:52008.

[RYZ] D. Ryabogin, V. Yaskin, A. Zvavitch, Harmonic analysis and uniqueness questions in convex geometry, In: Recent Advances in Harmonic Analysis and Applications (Eds. D. Bilyk et al.), Springer, New York, 2013, Proc. Math. Stat. 25, 327-337. MR 3066896.

[Sa] L. A. Santaló, Two characteristic properties of circles on a spherical surface (Spanish), Math. Notae 11 (1951), 73-78. MR 14,495c.

[Sch] R. Schneider, Convex Bodies: the Brunn-Minkowski Theory; Second expanded edition, Encyclopedia of Math. and its Appl., 44; 151, Cambridge Univ. Press, Cambridge, 1993; 2014. MR 94d:52007; 3155183.

[So] V. Soltan, Convex hypersurfaces with hyperplanar intersections of their homothetic copies, J. Convex Anal. 22 (2015), 145-159. MR 3346184.

[XMCh] G. Xiong, Y.-w. Ma, W.-s. Cheung, Determination of convex bodies from $\Gamma$-section functions, J. Shanghai Univ. 12 (3) (2008), 200-203. MR 2009d:52014.

[Y] V. Yaskin, Unique determination of convex polytopes by non-central sections, Math. Ann. 349 (2011), 647-655. MR 2012a:52011.

[YZh] V. Yaskin, N. Zhang, Non-central sections of convex bodies, http://arxiv.org/abs/1509. 08174.

MTA Alfréd RÉnyi Institute of Mathematics, H-1364 Budapest, Pf. 127, Hungary

http://www.renyi.mta.hu/ makai

Fakultät Für Mathematik, Technische Universität Chemnitz, D-09107 Chemnitz, Germany

E-mail address: makai.endre@renyi.mta.hu, martini@mathematik.tu-chemnitz.de 